\title{
L'usage de la catégorie de genre. Réponse de Thomas Laqueur à Annick Jaulin
}

Thomas Laqueur

\section{(2) OpenEdition \\ 1 Journals}

\section{Édition électronique}

URL : https://journals.openedition.org/clio/98

DOI : $10.4000 /$ clio.98

ISSN : 1777-5299

Éditeur

Belin

\section{Édition imprimée}

Date de publication : 1 avril 2002

Pagination : 209-211

ISBN : 2-85816-620-X

ISSN : $1252-7017$

Référence électronique

Thomas Laqueur, «L'usage de la catégorie de genre. Réponse de Thomas Laqueur à Annick Jaulin », Clio. Histoire, femmes et sociétés [En ligne], 15 | 2002, mis en ligne le 08 février 2005, consulté le 29 avril 2022. URL : http://journals.openedition.org/clio/98 ; DOI : https://doi.org/10.4000/clio.98 


\section{Varia}

\section{L'usage de la catégorie de genre. Réponse de Thomas Laqueur à Annick Jaulin}

Il est difficile, après avoir écrit tout un livre sur le thème de la différence sexuelle, d'apporter de nouvelles preuves pour défendre les points de vue qu'Annick Jaulin met en question dans le numéro 14 de CLIO, Histoire, Femmes et Sociétés. Elle a raison d'affirmer que l'usage que je fais de la catégorie de "genre" dans l'examen des sources prémodernes, est confus et peut-être inapproprié. J'ai moi-même essayé de le suggérer autant que faire se peut. Le mot luimême a longtemps été utilisé en anglais soit pour exprimer une distinction grammaticale ou une catégorie quelle qu’elle soit (un ensemble générique plutôt qu'une espèce), soit comme synonyme de sexe. Dans sa forme verbale transitive, gender signifiait aussi engendrer, produire naturellement. Dans ce sens, on peut dire qu'il y avait, chez Aristote, deux genres radicalement différents distingués par leur relation causale à la génération.

Ceci étant, l'usage moderne du mot date du féminisme des années soixantedix qui en fit un pôle du système dit de "sexe-genre» (l'anthropologue américaine R. Gayle fut, je crois, la première à employer la formule). Genre fait référence aujourd'hui aux caractéristiques culturelles qui ont été attribuées de manière contingente et diverse aux sexes biologiques. Le sexe, dans ce modèle, est supposé établi et donné une fois pour toutes; le genre ne l'est pas. Mon livre essayait de remettre en cause cette frontière en suggérant que le sexe comporte lui aussi

1 Claudine Leduc (dir.), CLIO, Histoire, Femmes et Sociétés, Festins de femmes, 14/2001.

CLIO, Histoire, Femmes et Sociétés, 2002, 15, pp. 209-211. 
un puissant composant culturel. S'il s'avère d'une manière ou d'une autre que les différences entre les sexes ne sont pas fondées, comme on le suppose, sur la biologie mais sur la métaphysique, nous, les modernes, avons un problème : en général nous n'acceptons pas la réalité transcendantale des catégories hiérarchiques utilisées par Aristote et beaucoup d'autres. Ainsi, le genre ne se situe pas sur le pôle opposé des catégories d'Aristote parce que nous n'acceptons pas l'ontologie sur laquelle elles se fondent. Il n'y a rien là auquel on puisse se référer. Nous pourrions, comme alternative, dire que celles-ci sont des catégories de genre, ce qui serait de toute évidence une proposition extravagante et a-historique. Aussi, j’ai essayé de suggérer, en guise de traduction, que les Anciens ont deux genres et un seul sexe (comme un double oxymore puisqu'un sexe signifie, par essence, aucun sexe et il n'y aurait alors rien pour établir le genre opposé). C'est précisément ce problème que la première partie de mon livre essayait de démêler.

Ceci nous ramène à la principale différence qui m’oppose à $\mathrm{A}$. Jaulin. Le cas de Galien semble assez clair. Les différences, dans une hiérarchie de l'être, se reflétaient dans l'anatomie : les positions opposées, interne et externe, d'organes qu'il considérait comme essentiellement les mêmes, reflétaient en toute vérité la classification du mâle et de la femelle à l'échelle de la chaleur vitale. Je suis absolument d'accord avec le fait qu'Aristote n'est pas aussi clair. Il parle d'une différence radicale entre les sexes qui semblerait invalider ma revendication d'un modèle «unisexe» dominant dans l'Antiquité. Mais A. Jaulin conviendra sûrement que la distinction faite par Aristote entre le mâle et la femelle est fondée, non sur la biologie, mais sur le rôle respectif qu'il leur attribue dans la génération : la cause formelle opposée à la cause matérielle. Celles-ci ne sont pas fondées, Aristote est bien en peine de le montrer, sur les substances concrètes de la génération, celles que nous pouvons observer. Le sperme visible n'est pas le résidu du grand "pool" qui a participé à la création d'une vie nouvelle ; le sang menstruel n'est pas la substance complémentaire. C'est le pneuma, le souffle, véhiculé par le sperme qui fait tout le travail et le pneuma n'est pas une substance biologique. Aristote ne fait pas non plus d'effort pour distinguer techniquement, de quelque façon que ce soit, les parties anatomiques de la génération. Alors oui, il y a deux sexes chez Aristote si nous entendons par différence sexuelle une forme de différence métaphysique. Mais ce n'est pas ce que nous voulons dire par sexe.

La problématique plus générale de mon livre est, bien sûr, la façon dont la biologie est parvenue à définir la différence sexuelle, rendant ainsi possible, la fabrication de deux sexes. Il s'agit de comprendre comment la biologie s'est substituée au cosmos, comment le monde que je décris dans les premières parties de 
mon livre a fait place au monde d'après les Lumières, étudié dans les chapitres suivants. Je n'avais pas l'intention de poser deux ensembles de catégories rigides pouvant aisément intégrer toutes les expressions de la différence sexuelle mais plutôt de fournir un modèle heuristique pour appréhender le sens de la grande transformation épistémologique et ontologique de la fin du XVII siècle au regard de notre sujet ${ }^{2}$. Je laisse à vos lectrices et lecteurs le soin de décider si mon histoire garde, entièrement ou partiellement, une valeur heuristique.

2 La rédaction de CLIO, Histoire, Femmes et Sociétés, rappelle que Yvonne Knibiehler avait abordé cette question dans un article pionnier paru en 1976 : «Les médecins et la nature féminine au temps du Code civil», Annales ESC, juillet-août 1976, pp. 824844. 